\title{
Determinants of glycemic and blood pressure control in type 2 diabetes patients: 606 outpatients diabetes cohort
}

Sabrina Coelli", Ariana Aguiar Soares, Ana Marina Moreira, Camila Kümmel Duarte, Luiza Barboza de Souza,

Themis Zelmanovitz, Sandra Pinho Silveiro

From 20th Brazilian Diabetes Society Congress

Porto Alegre, Brazil. 11-18 November 2015

\section{Background}

Diabetes mellitus (DM) complications are related to hyperglycemia, hypertension, smoking and lipids.

\section{Objectives}

The aim of this study was to evaluate the determinants of metabolic and blood pressure levels in type 2 diabetes.

\section{Materials and methods}

606 type 2 diabetes patients, outpatient Endocrine clinic, tertiary hospital, consecutively included between 20122014. Medical history, complete examination and laboratory evaluation performed (HbA1c, lipids, glomerular filtration rate -eGFR- and urinary albumin excretion-UAE).
Therapeutic targets defined according to American Diabetes Association: HbA1c $<7 \%$ ( $<8 \%$ if comorbidities); blood pressure $<140 / 90 \mathrm{mmHg}$, total cholesterol (TC) $<200 \mathrm{mg} / \mathrm{dL}$, HDL adjusted for gender and triglycerides (TG) $<150 \mathrm{mg} / \mathrm{dL}$. Approved by ethics committee $\left(\mathrm{n}^{\circ}\right.$ 140073); statistical analysis PASW 20.0.

\section{Results}

The mean age was $63 \pm 11$ yrs., $62 \%$ women, $86 \%$ white, $9 \%$ smokers, body mass index (BMI) $31 \pm 5 \mathrm{~kg} / \mathrm{m}^{2}$, median DM duration 16 yrs. Median eGFR was $91 \mathrm{ml} / \mathrm{min} /$ $1.73 \mathrm{~m}^{2}, 50 \%$ with UAE $>14 \mathrm{mg} / \mathrm{l}$. Diabetes treatment: $2 \%$ diet only, $67 \%$ insulin (alone or in combination with oral agents). Regarding therapeutic targets: $54 \%$ and $78 \%$ presented systolic (SBP) and diastolic blood pressure (DBP),

\begin{tabular}{|l|c|c|c|}
\hline & $\begin{array}{c}\text { Compensated glycemic } \\
\text { control group } \\
(\mathrm{N}=203)\end{array}$ & $\begin{array}{c}\text { Decompensated glycemic } \\
\text { control group } \\
(\mathrm{N}=403)\end{array}$ & $P$ \\
\hline Age (years) & $66 \pm 10$ & $62 \pm 11$ & $<0.001$ \\
\hline Women & $48 \%$ & $69 \%$ & $<0.001$ \\
\hline Black & $8 \%$ & $17 \%$ & 0.007 \\
\hline BMI (kg/m²) & $30 \pm 5$ & $31 \pm 5$ & 0.062 \\
\hline DM duration (years) & $15(2-46)$ & $16(2-52)$ & 0.095 \\
\hline HbA1c $(\%)$ & $6.6(4.3-7.9)$ & $9(7-15.2)$ & - \\
\hline UAE $(\mathrm{mg} / \mathrm{l})$ & $14.3(2.3-3402)$ & $13.7(2.3-5988)$ & 0.704 \\
\hline CKDEPI $\left(\mathrm{ml} / \mathrm{min} / 1.73 \mathrm{~m}^{2}\right)$ & $74(7-160)$ & $98(7-157)$ & $<0.001$ \\
\hline TG $(\mathrm{mg} / \mathrm{dl})$ & $132(28-1394)$ & $151(37-2742)$ & 0.013 \\
\hline TC $(\mathrm{mg} / \mathrm{dl})$ & $157(64-516)$ & $168(45-350)$ & $<0.001$ \\
\hline HDL $(\mathrm{mg} / \mathrm{dl})$ & $39(24-94)$ & $40(19-103)$ & 0.607 \\
\hline SBP $(\mathrm{mmHg})$ & $130(80-210)$ & $130(90-230)$ & 0.723 \\
\hline DBP $(\mathrm{mmHg})$ & $80(50-110)$ & $80(50-130)$ & 0.875 \\
\hline Insulin & $52 \%$ & $77 \%$ & $<0.001$ \\
\hline
\end{tabular}

Figure 1 Comparisons between compensated and decompensated control groups. 
respectively, within the recommendations. Median HbA1c was $8 \%$ (4.3-15.2\%), 36\% were below the target. TC, TG and HDL were at the target in, respectively, 79\%, $54 \%$ e $29 \%$ of the cases. The table shows the comparisons between compensated and decompensated glycemic control groups (Figure 1). Comparisons between gender did not show differences between age $(\mathrm{P}=0.61)$, $\mathrm{DM}$ duration $(\mathrm{P}=0.488)$ or blood pressure $(\mathrm{P}=0.117)$. However, women had higher BMI $(\mathrm{P}<0.001)$, worse lipid levels (TG: $\mathrm{P}=0.003$; TC: $\mathrm{P}<0.001)$ and glycemic control $(\mathrm{P}<0.001)$.

\section{Conclusion}

Two thirds of type 2 diabetes outpatients were above the recommended glycemic targets; obesity in women and black skin color were the main determinants for these findings.

Published: 11 November 2015

doi:10.1186/1758-5996-7-S1-A51

Cite this article as: Coelli et al:: Determinants of glycemic and blood pressure control in type 2 diabetes patients: 606 outpatients diabetes cohort. Diabetology \& Metabolic Syndrome 2015 7(Suppl 1):A51.
Submit your next manuscript to BioMed Central and take full advantage of:

- Convenient online submission

- Thorough peer review

- No space constraints or color figure charges

- Immediate publication on acceptance

- Inclusion in PubMed, CAS, Scopus and Google Scholar

- Research which is freely available for redistribution

Submit your manuscript at www.biomedcentral.com/submit
C Biomed Central 